Journal of Technology in Counselor Education and Supervision (ISSN 2692-4192)

Volume 1(1): 27 - 35

(C) 2021Journal of Technology in Counselor Education and Supervision

https://doi.org/10.22371/tces/004

\title{
Telehealth Best Practice: A Call for Standards of Care
}

\section{Donna Sheperis ${ }^{1} \&$ Arielle Smith ${ }^{2}$}

\begin{abstract}
Teletherapy is an approach to working with clients that mental health providers have used in some form for years. However, the onset of COVID forced the provision of therapy into teletherapy almost exclusively for a period of time. Currently, mental health providers conducting therapy virtually are not required to be trained in telehealth and are operating without consistent uniform standards of practice. This manuscript pulls from the current literature and guidelines across counseling, psychology, psychiatry, family therapy, and social work to provide a Proposed Standards of Practice for Telehealth. Such standards benefit mental health counselor by grounding practice in common aims and benefits out clients by creating a unified framework for providers engaging in telehealth with their clients. This manuscript provides a review of current literature around the provision of teletherapy and proposes a set of standards of care based on existing literature. In addition, gaps in current literature are addressed.
\end{abstract}

\section{Keywords}

telehealth; teletherapy; practice standards; COVID-19; mental health practice

The importance of technology as a modality for mental health treatment delivery has become increasingly clear. With the onset of COVID-19, the necessity of such services has risen to the forefront (da Silva \& Modesto, 2021). The benefits of teletherapy are expansive and have been established through a large body of literature. Patients and providers are generally satisfied with telehealth services and the demand to close the treatment gap by implementing technologies is high among both parties (Hubley et al., 2016). Advantages include improved access, availability, and flexibility of services, enhanced communication between provider and client, cost-effectiveness, reduction of geographic and mobility barriers, and increased client empowerment (Stoll et al., 2020). Despite the support for teletherapy in the literature, providers have been hesitant to implement technology into their practices or to refer clients to teleproviders (Perle et al., 2014). However, a global pandemic has forced this issue to the surface. While there certainly are ethical, legal, privacy, delivery, and safety concerns specific to conducting teletherapy, and proper training should

$1 \quad$ Palo Alto University, Palo Alto, CA, USA

2 Washington University St. Louis, St. Louis, MO, USA address these concerns, we recognize that this is true of all modalities. Mental health providers can practice remotely and do it well, with an understanding of best practice. As such, teletherapy can best be framed as a delivery model, not as a new clinical specialty (Cooper et al., 2019).

Although apprehension among mental health counselors and the lack of training available has dissuaded many practitioners from adopting teletherapy in the past, the COVID-19 crisis spurred a rapid migration of mental health providers from in-person to online services. The state of the world after COVID-19 is still unknown, but a return to in-person therapy as the default is not necessarily the best-case or only scenario. In fact, telemental health services are likely to play a key role in addressing the psychological impacts of the pandemic (Zhou et al., 2020). Furthermore, because of its many benefits, teletherapy should continue to be implemented as a primary service delivery option for mental health providers and their clients once the self-isolation ceases (Fejit, et al., 2020). In order to successfully disseminate effective telemental health services,

Corresponding Author: Donna Sheperis, PhD, Palo Alto University, 1791 Arastradero Road, Palo Alto, CA 94304 email: dsheperis@paloaltou.edu 
there needs to be uniform standards of care across the mental health professions. Currently, the onus is on individual practitioners to largely teach themselves how to transition their services online. Some providers can look toward their professional organizations that have published their own teletherapy guidelines, but these are not professional standards nor are they uniform or accessible for all providers. For example, the National Board for Certified Counselors (NBCC, 2016) offers a policy document that applies only to Nationally Certified Counselors (NCCs). To assist, entities such as the American Mental Health Counselors Association (AMHCA) have begun to aggregate these individual policies and guides on their member website (2020). However, the individual practitioner is still left to review the myriad of policies in an effort to determine standard best practice.

Due to the lack of consistency and uniformity, the purpose of this article is to address the gap in telemental health implementation and to provide recommendations for future research and publications. Within this manuscript, we propose a comprehensive list of practice standards based on a systematic search of existing literature and policy. Finally, we will note current deficits in existing research and discuss opportunities for further work to improve the practice of telehealth within the field of mental health care.

\section{Method}

The authors conducted a review of literature across the professions of counseling, psychology, psychiatry, and social work related to telehealth services. Specifically, the researchers searched Ebscohost; MEDLINE; Google Scholar; and public facing professional organization documents including those from counseling, psychology, social work, and family therapy; as well as suggested guidelines from the Department of Defense, American Telemedicine Association, the National Board for Certified Counselors, the American Counseling Association, the American Mental Health Counselors Association, the American Psychiatric Association, and the American Psychological Association. Disagreements were resolved by face-to-face discussion and brought to the research team within a university eclinic for consultation. Our search revealed 12 areas that have been addressed via research or policy across the telehealth disciplines. Each of the 12 areas was further supported by 2-4 recommendations which were also brought to the larger eclinic team for consultation following independent author review. What follows is how the literature shaped each area and recommendation being proposed.

\section{Best Practice in Telehealth}

In this section, we will address twelve areas that would be essential to best practice. Following the description of the area, we will also offer a proposed standard of practice.

\section{Training}

As soon as the various shelter in place mandates began, therapists had to scramble for alternative ways to meet client needs. If face to face work was no longer possible or safe, what could we do to continue treatment plans that were in progress as well as see new patients suffering due to the many challenges of the crisis? We quickly learned that there was a high need for training. Often it has been the lack of training or exposure that has kept practitioners from engaging in telehealth. We know that the majority of practitioners become more comfortable with the use of telehealth following some form of professional education (Perle et al., 2014).

Clearly there are unique clinical considerations when providing teletherapy that require training. Most clinical training programs that prepare therapists for licensure do not cover the provision of telemental health care (Mace, Boccanelli, \& Dormond, 2018). In addition, most clinicians saw telehealth as a useful form of service delivery even before it became virtually required due to COVID-19 (Glueckauf et al., 2018).

Proposed Standard I: Providers shall become appropriately trained in the specific skills required to administer teletherapy and continue with ongoing education.

\section{Platform Selection}

The variety of potential platforms to conduct telehealth services can be overwhelming to beginning practitioners. When making the decisions during non-pandemic times, practitioners can take their time vetting many available solutions. In a pandemic, many of us are pushing forward with platforms with which we are initially unfamiliar but can be verified as compliant with the Health Insurance Portability and Accountability Act (HIPAA) of 1996; provide synchronous and asynchronous options; and offer technological support for both the provider and the client.

\section{Synchronous Platforms}

Synchronous videoconferencing platforms that have been specifically designed for teletherapy often have features that are convenient for therapy, are consistent with HIPAA regulations, and provide Business Associate Agreements (Cooper et al., 2019). Recommendations for videoconferencing software include the ability to display static pictures/graphs, record sessions, share information through computer files, use with minimal training, and capture nonverbal cues (Yellowlees et al., 2010). To appropriately use synchronous platforms, 
practitioners must attend to the fact that eye contact, body position, facial expressions, and other forms of nonverbal communication are just as relevant in videoconferencing teletherapy as in face-to-face therapy (Henry et al., 2017). Not all digital platforms are just for synchronous or video work. There also need to be methods of interacting with patients asynchronously.

\section{Asynchronous Platforms}

Asynchronous platforms allow therapists and clients to continue the work of therapy outside of a video session. Application, or app, based asynchronous platforms can be used by the patient outside of sessions but in conjunction with sessions. These apps include those that allow for self-monitoring between sessions; provide opportunities for assessment; and provide psychoeducation and coping skills training (Armstrong et al., 2017). Other apps may be used by patients on their own as they are designed solely for self-help. Some of the apps are also helpful with crisis support (Armstrong et al., 2017).

Practitioners need to be aware that while there are thousands of mental health applications available, most are not evidenced-based or clinically validated (Wang et al., 2018). As such, careful consideration must be applied when recommending applications to clients. Before recommending an application, the therapist will need to identify the level of support for its efficacy and clinical utility by reviewing any research conducted using the particular app based intervention. (Armstrong et al., 2017). In addition, therapists should consider how applications will fit into the patient's overall treatment plan.

Proposed Standard II: Providers shall review the variety of teletherapy platforms and mental health applications available and select mediums that are secure, evidence-based, and support clinical integration.

\section{Creating an Office for Virtual Work}

When conducting telehealth services, whether in a pandemic or as part of an intentional practice, De Weger and colleagues (2013) suggest that providers consider their virtual space as they make the transition to online care. Specifically, clinicians should conduct sessions in a well-lit room that is free of backlighting. Prior to conducting a session, the provider should test equipment to ensure that the camera gaze angle emulates eye contact; minimize background noise; and ensure that sessions are conducted in a private space (De Weger et al., 2013).

In addition to their virtual office set up, providers should help guide their patients through the process of setting up a space that is private and distractionfree (Morland et al., 2015). An initial conversation with patients to orient them to videoconferencing and working through technology or privacy barriers is recommended as well.

Proposed Standard III: Providers shall consider the physical environment that they practice in and that of their clients in order to collaborate on optimal set-ups for videoconferencing.

\section{Legal Concerns}

Before starting telehealth, therapists are asked to become familiar with federal regulations, including but not limited to, the Health Information Portability and Accountability Act (HIPAA 1996), the Health Information Technology for Economic and Clinical Health Act (HITECH Act 2009), and the Family Educational Rights and Privacy Act (FERPA 1974) (Lustgarten \& Elhai, 2018). With the addition of COVID-19, there have been changes in the federal laws specifically allowing exceptions for telehealth reimbursement and tele supervision of practitioners in training. While a complete review of those laws is outside the scope of this manuscript, the idea that laws are constantly changing at the federal and state level, and that the onset of a pandemic has created even greater changes, is essential to providing telehealth services.

In general, states require providers to hold an active professional license in the state that the client is physically located during sessions (Shore et al., 2018). However, some exceptions exist due to COVID-19 that must be explored on a state by state level. In addition, there are a number of states without laws that specifically regulate teletherapy. If this is the case, mental health providers should refer to the regulations set by their state licensing board (Miller, 2020; Telehealth Certification Institute, 2016; Joint Task Force for the Development of Telepsychology Guidelines for Psychologists, 2013). At present, there is not a system that allows all providers to practice across state lines, so many mental health providers may not practice interjurisdictionally unless they hold multiple state licenses and plan to only treat clients located in the states where a license is held (Miller, 2020). It is recommended that clinicians consult with a lawyer who specializes in healthcare policy to navigate these changes (Lustgarten \& Elhai, 2018).

Proposed Standard IV: Providers shall thoroughly review and always comply with the federal and state laws dictating the regulations for conducting teletherapy.

\section{Accessibility}

Providing accessible services is the responsibility of practitioners whether in person or virtually. Specific to technological accessibility, providers understand that cultural variables including race, ethnicity, age, 
location, language, education, socioeconomic status, and disability can be limiting factors on the client's ability to participate in teletherapy (Cooper et al., 2019). Specifically, we want to eliminate barriers to promote equity of access by selecting platforms/applications that do not have additional fees for clients, that are available on many types of electronic devices, and that have features to enhance physical accessibility. We also know that clients seeking teletherapy will have different prior experiences with technology, contributing to varied comfort levels, concerns about confidentiality, and communication styles (Shore et al., 2006). Some clients may be more comfortable with phone calls and the portability of mobile phones also provides the opportunity for clients to engage in behavioral exercises in various locations (Yuen et al., 2012). It is the role of the mental health counselor to empower each client to make a personal choice about whether or not to accept the technology used in the teletherapy practice (Armstrong et al., 2017).

Proposed Standard V: Providers shall maximize the accessibility of services for all populations and consider the culture of each client when assisting them in the uptake of technologies.

\section{Working with Multiple Clients}

Many providers specialize in work with couples and families. That work is still possible during a pandemic and couple and family therapy can actually be enhanced by telehealth practices (Doss et al., 2017). Teletherapy is well-suited to accommodate the coordination of multiple schedules and reduces the costs associated with missing work or school. Asynchronous platforms designed for couples and families eliminate time barriers and allow multiple people to participate in therapy with ease. Teletherapy that is delivered in the clients' homes may provide more insight into couple or family dynamics and increase the ecological validity of the intervention strategies (Doss et al., 2017). Mental health counselors will need to become familiar with the theories and frameworks that detail the impact of technology on couple and family relationships and address these factors in treatment accordingly (Blumer et al., 2015).

In addition to couple and family work, group work is also possible via telehealth. Technology can be used in group therapy to provide relatively low-cost interventions that help clients overcome social isolation (Banbury et al., 2018), something that has increased during COVID. We know that teletherapy facilitates bonding and cohesiveness at comparable levels to in-person group therapy and is acceptable by clients (Banbury et al., 2018),

Proposed Standard VI: Providers working with couples, families, and groups shall consider how teletherapy technology can be used successfully with multiple parties.

\section{Screening}

Screening for appropriateness of a treatment modality has long been part or the therapeutic responsibilities. For telehealth practice, it is essential to determine if potential clients possess certain characteristics which may make them unsuitable candidates for the teletherapy services provided and make referrals accordingly (Morland et al., 2015). Specifically, clients who do not have access to the required technologies, who reject the technologies during the informed consent process, or who have severe psychosis, mood dysregulation, suicidal ideation, homicidal tendencies, substance use disorder, cognitive impairment, or sensory impairment may not be best served by teletherapy. It is also necessary to use age-appropriate strategies when providing services to children, adolescents, or older adults (NBCC, 2016; Richardson et al., 2009).

Proposed Standard VII: Providers shall ensure that the teletherapy services they provide meet the needs of the specific populations they work with.

\section{Informed Consent}

Similar to face to face work, providers need to obtain informed consent through a process that details benefits, risks, and expectations of telehealth. Benefits include enhanced access to services; fewer scheduling and transportation barriers; ability to monitor change in real time; and cost effectiveness (Reamer et al., 2017). Risks specific to this type of work include lack of technology; technology failure; and potential data breaches (Reamer et al., 2017). Emergency procedures, billing practices, and communication policies should also be included (Cooper et al., 2019). All of these elements must be share in language that is understandable to clients with varied education levels and be aware of the linguistic, cultural, and socioeconomic barriers that might interfere with comprehension (Joint Task Force for the Development of Telepsychology Guidelines for Psychologists, 2013).

Proposed Standard VIII: Providers shall obtain voluntary informed consent through a process that details the benefits, risks, and expectations specific to teletherapy.

\section{Data Security}

In person practices have their own challenges with data storage and security. When conducting telehealth, those challenges are even more acute. Specifically, data transmission, storage, and disposal methods as well as the procedures by which clients and appropriate organizations will be notified if there is a data breach 
must be outlined in the informed consent (NBCC, 2016; Joint Task Force for the Development of Telepsychology Guidelines for Psychologists, 2013). The use of HIPAA compliant software that includes advanced security features such as password protection, two-factor authentication, and antivirus, antimalware, and firewall software updates is recommended. (Lustgarten \& Elhai, 2018). Practitioners can then work with their patients to ensure that the personal devices they use to communicate are secure (Lustgarten \& Elhai, 2018). These practices should result in a plan to mediate and address any risks associated with data security in telehealth.

Proposed Standard IX: Providers shall assess and have plans to mediate the specific risks associated with data security.

\section{Emergency Management}

Emergency and crisis management is common in mental health practice. When working virtually with patients, there are a few steps to increase our ability to help clients in crisis. At intake, practitioners should take care to verify the client's identity and contact information; verify their current location, residential address, and where they will be during sessions; gather contact information for any other healthcare providers; develop expectations for contact between sessions during emergency and non-emergency situations; and, where needed, create and document a safety plan (Shore et al., 2018).

Providers will also need to be familiar with all duty to warn and protect requirements that fit the state as well as civil commitment policies and laws (Kramer et al., 2015). Given that providers and patients may be in different cities, it is essential to collect the contact information of the local law enforcement and emergency services and establish a plan to collaborate with them if necessary (Shore et al., 2007). Finally, providers and patients should develop a plan for navigating service interruptions that might occur due to technical issues or patient's disconnecting from the service while in crisis (Kramer et al., 2015).

Proposed Standard X: Providers shall create an emergency management protocol with each individual client upon intake and proceed with ongoing modification as necessary.

\section{Boundaries}

During the initial session, including informed consent, and throughout the virtual relationship, it is the responsibility of the clinician to set and maintain boundary expectations to protect both the provider and the client. Considerations may include the business hours when clients can expect to reach providers; within what time period clients can expect a response; policies to address situations in which clients inappropriately cross contact boundaries; and who clients should contact during emergency situations that occur outside of defined business hours (Lustgarten \& Elhai, 2018). Communicate electronically with clients only after they have consented and do not post any potentially identifying information about clients on professional and/ or personal social media platforms (Association of Social Work Boards International Technology Task Force, 2015).

Regarding social media presence and boundaries in telehealth, providers are advised to take precautions to separate personal and professional social media use. They should use a designated professional email to set up the professional social media accounts (Kramer et al., 2015). Privacy settings should be reviewed on all personal accounts to prevent clients from following personal accounts (Lustgarten \& Elhai, 2018). Understandable, providers are asked to refrain from posting anything personal on professional social media accounts and blogs in order to avoid boundary confusion and inappropriate dual relationships (Association of Social Work Boards International Technology Task Force, 2015). Finally, recall that shared cultural factors may increase the likelihood of an unintended encounter with clients online and it is essential to establish a procedure for addressing such instances (Association of Social Work Boards International Technology Task Force, 2015).

Proposed Standard XI: Providers shall consider the unique factors involved in setting professional boundaries in online spaces.

\section{Assessment}

One of the more challenging practices in telehealth is in the area of assessment. While providers may be accustomed to conducting assessments on paper or face to face, some assessments, like self-report questionnaires, are particularly conducive to delivering online. In addition, there are some possible advantages to conducting self-report questionnaire assessments online including greater honesty and disclosure, increased outreach capabilities, convenience, and efficiency (Yuen et al., 2012). While structured clinical interviews require some planning to successfully adapt to online administration, it is definitely feasible to do in telehealth. Any environmental factors that can compromise the integrity of the assessment should be discussed and addressed with clients, including distractions in the client's space (Joint Task Force for the Development of Telepsychology Guidelines for Psychologists, 2013). Just as is required for using any assessment, appropriate training is needed to then use the assessment in a telehealth practice. Conducting teletherapy requires specialized training in order to 
ensure the integrity of the assessment and treatment processes within the unique considerations of the online environment (Titov et al., 2019).

Proposed Standard XII: Providers shall adapt assessment and treatment processes to the teletherapy environment to uphold the integrity of theoretical approaches when services are delivered virtually.

In Figure 1, we have outlined the aformentioned proposed standards of practice for telehealth and provided key tasks to meet the standards.

Implications and Limitations of Standards of Care Research

Having standards of care for telehealth can offer mental health providers and their clients opportunities for successful work within an under studied realm of service provision. In addition, a recognized set of standards can provide a framework for teaching and training of mental health providers. Finally, these standards help practitioners offer ethical and competency based services to the public. By extension, such standards help establish groundwork for the supervision of such services.

We recognize the limitations of a literature review to establish standards of care. We also recognize that the current literature must serve as a starting point for such standards which can evolve as our understanding of telehealth care evolves. For the development of the standards presented in this manuscript, we discovered a number of areas lacking robust levels of empirical research. Specifically, the impact of teletherapy training such as continuing education courses has not been studied as it relates to client outcome. While guidance does exist related to choosing platforms and apps (American Psychiatric Association, 2020), the

Figure 1. Proposed Standards of Practice for Telehealth

\section{Proposed Standards of Practice for Telehealth}

Standard I: Providers shall become appropriately trained in the specific skills required to administer teletherapy and continue with ongoing education.

a. Recognize that lack of training in conducting teletherapy and insufficient knowledge about the efficacy of teletherapy deters providers from practicing teletherapy.

b. Understand that there are clinical considerations unique to delivering teletherapy that must be learned through specialized training.

c. Identify the discrepancy between providers' interest in teletherapy and their actual ability to conduct teletherapy due to insufficient training.

Standard II: Providers shall review the variety of teletherapy platforms and mental health applications available and select mediums that are secure, evidence-based, and support clinical integration.

a. Review asynchronous and synchronous options and decide which type(s) of technical tool(s) to use in practice.

b. Asynchronous methods may be used in conjunction with therapy or external to therapy

c. Consider synchronous methods to mirror the experience of traditional therapy

Standard III: Providers shall consider the physical environment that they practice in and that of their clients in order to collaborate on optimal set-ups for videoconferencing.

a. Apply recommendations for the provider's physical environment

b. Guide clients through the process of setting up a space that is private and distraction-free

c. Begin with an initial conversation with clients to orient them to the videoconferencing platform and work through technological or personal barriers that may interfere with their ability to use the technology comfortably.

Standard IV: Providers shall thoroughly review and always comply with the federal and state laws dictating the regulations for conducting teletherapy.

a. Become familiar with federal regulations

b. Comply with all state laws related to practicing therapy in general and those that are specific to conducting teletherapy.

Standard V: Providers shall maximize the accessibility of services for all populations and consider the culture of each client when assisting them in the uptake of technologies.

a. Understand that cultural variables including race, ethnicity, age, location, language, education, socioeconomic status, and disability can be limiting factors on the client's ability to participate in teletherapy

b. Assess each clients' relationships with technology and modify treatment accordingly to improve outcomes 
c. Empower each client to make a personal choice about whether or not to accept the technology used in the teletherapy practice.

Standard VI: Providers working with couples, families, and groups shall consider how teletherapy technology can be used successfully with multiple parties.

a. Recognize that technology has the potential to enhance couple and family therapy

b. Become familiar with the theories and frameworks that detail the impact of technology on couple and family relationships and address these factors in treatment accordingly

c. Understand that technology can be used in group therapy to provide relatively low-cost interventions that help clients overcome social isolation

Standard VII: Providers shall ensure that the teletherapy services they provide meet the needs of the specific populations they work with.

a. Determine if potential clients possess certain characteristics which may make them unsuitable candidates for the teletherapy services provided and make referrals accordingly

b. Use age-appropriate technologies and practices if working with children, adolescents, or older adults

c. Exercise cultural awareness and identify the unique characteristics of populations that may influence virtual treatment

Standard VIII: Providers shall obtain voluntary informed consent through a process that details the benefits, risks, and expectations specific to teletherapy.

a. Discuss possible benefits specific to teletherapy including enhanced access

b. Discuss possible risks specific to teletherapy including cost of technology; technology failure; and potential for data breech

c. Outline expectations for how the technology will be integrated into clinical practice

d. Ensure that the language in the informed consent document is understandable to clients with varied education levels and be aware of the linguistic, cultural, and socioeconomic barriers that might interfere with comprehension

Standard IX: Providers shall assess and have plans to mediate the specific risks associated with data security.

a. Be specific in the informed consent document about risk and risk management

b. Use a HIPAA compliant software with advanced security features

c. Work with clients to ensure that the personal devices they use to communicate are secure

Standard X: Providers shall create an emergency management protocol with each individual client upon intake and proceed with ongoing modification as necessary.

a. Verify client location and emergency information

b. Discuss expectations for contact between sessions during emergency and non-emergency situations.

c. Become familiar with the jurisdiction's civil commitment laws and duty to warn and protect requirements

Standard XI: Providers shall consider the unique factors involved in setting professional boundaries in online spaces.

a. Establish electronic communication boundaries and expectations

b. If maintaining a professional social networking presence, take precautions to separate personal and professional social media use.

Standard XII: Providers shall adapt assessment and treatment processes to the teletherapy environment to uphold the integrity of theoretical approaches when services are delivered virtually.

a. Understand that some psychological assessments, like self-report questionnaires, are particularly conducive to delivering online

b. Understand that structured clinical interviews require some thought to successfully adapt to online administration

c. Receive appropriate training for any assessment being used in a telehealth setting 
profession is missing guidance on how to successfully integrate such apps into practice. Further, the lack of evidence-based protocols demonstrated by some apps could dissuade providers from using this technology.

The current literature is able to provide us with the need to ensure that clients engaging in telehealth are in a private space. However, we do not have a clear understanding how providers can help clients troubleshoot if such a space is not available. Barriers to access and ethical considerations could be evaluated in light of space, technology, and privacy for clients. A final gap that was evident in the development of these proposed standards was the need for a utilitarian, case study approach to help mental health counselors determine how they can apply best practice standards in their own provision of telehealth care. While this manuscript is theoretical based on an analysis of the literature, a practical manuscript that showed how to apply such concepts would be an excellent complement.

\section{Conclusion}

Overall, as the need for telehealth increases due to a worldwide pandemic as well as a greater interest in alternative provision of traditional health and mental health care, providers are in need of standards to drive the process. Guidance for mental health counselors in selecting and using platforms; screening and preparing clients for telehealth care; managing risk, legal, and ethical concerns, and maintain competency are just a few of the areas where therapists are looking for guidance to meet best practice. This manuscript reviewed the existing literature across telehealth disciplines and generated a set of proposed standards for consideration. The anticipated outcome is that mental health providers will find a useful set of care standards that can be further developed as the research base grows. are more likely to see stories from sources they have already engaged with in the past (DeMers, 2016). For professional counseling organizations that share updates and information with followers on Facebook, the

\section{References}

American Mental Health Counselors Association (2020). Telehealth resources. https://www.amhca.org/publications/ practiceguidelines/coronavirus/telehealth

American Psychiatric Association (2020). APP advisor: An American Psychiatric Association initiative. https://www. psychiatry.org/psychiatrists/practice/mental-health-apps

Armstrong, C. M., Edwards-Stewart, A., Ciulla, R. P., Bush, N. E., Cooper, D. C., Kinn, J. T., Pruitt, L. D., Skopp, N. A., Blasko, K. A., \& Hoyt, T. V. (2017). Department of Defense mobile health practice guide (4th ed.). Defense Health Agency Connected Health, U.S. Department of Defense.
Association of Social Work Boards International Technology Task Force. (2015). Model regulatory standards for technology and social work practice. https://www.aswb.org/ wp-content/uploads/2015/03/ASWB-Model-RegulatoryStandards-for-Technology-and-Social-Work-Practice.pdf

Banbury, A., Nancarrow, S., Dart, J., Gray, L., \& Parkinson, L. (2018). Telehealth interventions delivering home-based support group videoconferencing: systematic review. Journal of medical Internet research, 20(2), e25.

Blumer, M. L., Hertlein, K. M., \& VandenBosch, M. L. (2015). Towards the development of educational core competencies for couple and family therapy technology practices. Contemporary Family Therapy, 37(2), 113-121.

Cooper, S. E., Campbell, L. F., \& Smucker Barnwell, S. (2019). Telepsychology: A primer for counseling psychologists. The Counseling Psychologist, 47(8), 1074-1114.

da Silva, F. C. T., \& Neto, M. L. R. (2021). Psychiatric symptomatology associated with depression, anxiety, distress, and insomnia in health professionals working in patients affected by COVID-19: A systematic review with metaanalysis. Progress in Neuro-Psychopharmacology \& Biological Psychiatry, 104, N.PAG. https://doi-org.paloaltou.idm.oclc.org/10.1016/j.pnpbp.2020.110057

De Weger, E., Macinnes, D., Enser, J., Francis, S. J., \& Jones, F. W. (2013). Implementing video conferencing in mental health practice. Journal of Psychiatric and Mental Health Nursing, 20(5), 448-454.

Doss, B. D., Feinberg, L. K., Rothman, K., Roddy, M. K., \& Comer, J. S. (2017). Using technology to enhance and expand interventions for couples and families: Conceptual and methodological considerations. Journal of Family Psychology, 31(8), 983-993.

Feijt, M., de Kort, Y., Bongers, I., Bierbooms, J., Westerink, J., \& IJsselsteijn, W. (2020). Mental health care goes online: Practitioners' experiences of providing mental health care during the covid-19 pandemic. Cyberpsychology, Behavior, and Social Networking. https://doi-org.paloaltou.idm.oclc.org/10.1089/cyber.2020.0370

Glueckauf, R. L., Maheu, M. M., Drude, K. P., Wells, B. A., Wang, Y., Gustafson, D. J., \& Nelson, E. L. (2018). Survey of psychologists' telebehavioral health practices: Technology use, ethical issues, and training needs. Professional Psychology: Research and Practice, 49(3), 205-219.

Henry, B. W., Block, D. E., Ciesla, J. R., McGowan, B. A., \& Vozenilek, J. A. (2017). Clinician behaviors in telehealth care delivery: a systematic review. Advances in Health Sciences Education, 22(4), 869-888.

Joint Task Force for the Development of Telepsychology Guidelines for Psychologists. (2013). Guidelines for the practice of telepsychology. American Psychologist, 68(9), 791-800.

Kramer, G. M., Kinn, J. T., \& Mishkind, M. C. (2015). Legal, 
regulatory, and risk management issues in the use of technology to deliver mental health care. Cognitive and Behavioral Practice, 22(3), 258-268.

Lustgarten, S. D., \& Elhai, J. D. (2018). Technology use in mental health practice and research: Legal and ethical risks. Clinical Psychology: Science and Practice, 25(2), e12234.

Mace, S., Boccanelli, A., \& Dormond, M. (2018). The use of telehealth withing behavioral health settings: Utilization, opportunities, and challenges. University of Michigan School of Public Health: Behavioral Health Workforce Research Center. https://behavioralhealthworkforce.org/wpcontent/uploads/2018/05/Telehealth-Full-Paper_5.17.18clean.pdf

Miller, J. (2020). Impact of the coronavirus outbreak: What you need to know on providing tele-mental health services. https://www.amhca.org/blogs/joel-miller/2020/03/18/ impact-of-the-coronavirus-outbreak-what-you-need-t

Morland, L. A., Poizner, J. M., Williams, K. E., Masino, T. T., \& Thorp, S. R. (2015). Home-based clinical video teleconferencing care: Clinical considerations and future directions. International Review of Psychiatry, 27(6), 504-512.

National Board of Certified Counselors. (2016). Policy regarding the provision of distance professional services. https://www.nbcc.org/Assets/Ethics/NBCCPolicyRegarding PracticeofDistanceCounselingBoard.pdf

Perle, J. G., Burt, J., \& Higgins, W. J. (2014). Psychologist and physician interest in telehealth training and referral for mental health services: An exploratory study. Journal of Technology in Human Services, 32(3), 158-185.

Reamer, F. G., Barsky, A. E., Comer, M. J., Groshong, L. W., Hobdy, D. M., Hymans, D. J., Monahan, M. J., \& Regan, J. A. R., (2017). Technology standards in social work practice. National Association of Social Workers, Association of Social Work Boards, Council on Social Work Education, Clinical Social Work Association. https://www. socialworkers.org/includes/newincludes/homepage/PRABRO-33617.TechStandards_FINAL_POSTING.pdf

Richardson, L. K., Christopher Frueh, B., Grubaugh, A. L., Egede, L., \& Elhai, J. D. (2009). Current directions in videoconferencing tele-mental health research. Clinical Psychology: Science and Practice, 16(3), 323-338.

Shore, J. H., Savin, D. M., Novins, D., \& Manson, S. M. (2006). Cultural aspects of telepsychiatry. Journal of Telemedicine and Telecare, 12(3), 116-121.

Shore, J. H., Hilty, D. M., \& Yellowlees, P. (2007). Emergency management guidelines for telepsychiatry. General Hospital Psychiatry, 29(3), 199-206.

Shore, J. H., Yellowlees, P., Caudill, R., Johnston, B., Turvey, C., Mishkind, M., Krupinski, E., Myers, K., Shore, P., Kaftarian, E., \& Hilty, D. (2018). Best practices in videoconferencing-based telemental health. The American Psychiatric Association, The American Telemedicine Association.
https://www.psychiatry.org/File\%20Library/Psychiatrists/ Practice/Telepsychiatry/APA-ATA-Best-Practices-in-Videoconferencing-Based-Telemental-Health.pdf

Telehealth Certification Institute (2016). States rules and regulations. https://telementalhealthtraining.com/ states-rules-and-regulations

Titov, N., Hadjistavropoulos, H. D., Nielssen, O., Mohr, D. C., Andersson, G., \& Dear, B. F. (2019). From research to practice: Ten lessons in delivering digital mental health services. Journal of Clinical Medicine, 8(8).

Wang, K., Varma, D. S., \& Prosperi, M. (2018). A systematic review of the effectiveness of mobile apps for monitoring and management of mental health symptoms or disorders. Journal of Psychiatric Research, 107, 73-78.

Yellowlees, P., Shore, J., \& Roberts, L. (2010). Practice guidelines for videoconferencing-based telemental health. Telemedicine and e-Health, 16(10), 1074-1089.

Yuen, E. K., Goetter, E. M., Herbert, J. D., \& Forman, E. M. (2012). Challenges and opportunities in internet-mediated telemental health. Professional Psychology: Research and Practice, 43(1).

\section{Author Notes:}

Donna S. Sheperis, https://orcid.org/0000-0001-5732-8697 